COLORECTAL CANCER

\title{
Association between family history and mismatch repair in colorectal cancer
}

\author{
R P Coggins, L Cawkwell, S M Bell, G P Crockford, P Quirke, P J Finan, D T Bishop
}

Gut 2005;54:636-642. doi: 10.1136/gut.2003.017517

See end of article for authors' affiliations

Correspondence to

Professor D T Bishop,

Genetic Epidemiology

Division, Cancer Research

UK Clinical Centre, Cancer

Genetics Building, $\mathrm{St}$

James's University

Hospital, Beckett St, Leeds

LS9 7TF, UK; Tim.Bishop@

cancer.org.uk

Revised version received 30 September 2004

Accepted for publication

26 October 2004
Background and aims: Germline mutations in mismatch repair (MMR) genes cause a greatly increased risk of cancer of the gastrointestinal and female reproductive tracts (hereditary non-polyposis colorectal cancer (HNPCC)). Loss of MMR expression is common in colorectal cancer (CRC) overall. Such loss is assumed to be acquired predominantly, although a population of CRC cases will include individuals with unrecognised MMR mutations. This study examines the association between MMR gene expression and family history of cancer among the CRC population.

Methods: Individuals with CRC were identified from two well characterised populations: (1) consecutive hospital patients $(n=644)$ and $(2)$ a population based cases series $(n=249)$. CRC was examined for expression of $\mathrm{hMLH1}$ and $\mathrm{hMSH} 2$ using immunohistochemistry, and expression was related to family history using logistic regression.

Results: hMLH1 and hMSH2 expression was assessed in 732 CRCs with $8 \%$ showing loss of expression. No association was seen overall for $\mathrm{hMLH} 1$ or $\mathrm{hMSH} 2$ expression and family history of CRC. Loss of hMSH2 was predicted by family history of extracolonic cancer (odds ratio (OR) 5.78 (95\% confidence interval (Cl) 0.95-35.18)) and family history suggestive of HNPCC (OR 27.84 (95\% Cl 4.37-177.56)). Loss of hMLH1 was not predicted by family history of extracolonic cancer or a family history suggestive of HNPCC but was for a family history of at least two affected relatives (OR 4.88 (95\% Cl 1.25-19.03)).

Conclusions: Individuals with hMSH2 deficient CRC in the general population exhibit a family history and other characteristics suggestive of HNPCC, and may carry germline MMR mutations. Loss of hMLHI is only associated with a strong family history of extracolonic cancer at older ages, suggesting a novel mechanism of susceptibility.
A pproximately 5\% of all colorectal cancers (CRCs) are thought to arise as a result of familial adenomatous polyposis or hereditary non-polyposis colorectal cancer (HNPCC). Although these dominantly inherited cancer susceptibility syndromes account for only a small proportion of CRCs in general, they are of significance because of their associated high cancer risk. Furthermore, their study has greatly increased our understanding of the molecular events driving CRC development. In comparison, familial aggregation of CRC within families in the absence of a recognisable inherited trait is common. Numerous studies have noted that $10-20 \%$ of individuals affected by CRC report a close family member also affected by the disease. ${ }^{12}$ It is estimated that close relatives of individuals affected by CRC have a two- to fivefold increased risk of developing CRC themselves. ${ }^{3}$ Cancer risk increases with the number of first degree relatives affected, and is particularly high if a first degree relative develops CRC under the age of 45 years. ${ }^{45}$ Similar studies have reported a close association between family history of CRC and the development of colorectal adenomas. ${ }^{67}$

HNPCC is characterised by the development of cancer affecting the gastrointestinal, female reproductive, and urinary tracts. ${ }^{8}$ Cancers often occur at a young age, are often multiple, and when arising in the colon are frequently proximal in location. However, there is no characteristic phenotype associated with HNPCC, and diagnosis is dependent on recognition of a strong family history suggestive of dominant inheritance.

HNPCC is known to result from inherited deficiency of mismatch repair (MMR). Human MMR is mediated by a number of genes, including hMLH1, hMSH2, hMSH6, hPMS1, hPMS2, hMLH3, and hMSH3. ${ }^{9-11}$ Together these genes are responsible for recognising and initiating repair of mismatched base pairs. Failure of any component of MMR has the potential to allow incorporation of unrecognised base pair mismatches into the genome. This process may be sufficient to affect subsequent expression of genes that are essential to normal cell function, regulation, and growth. ${ }^{12}$ Mismatching of base pairs commonly occurs through somatic mutation in microsatellite DNA sequences which consist of simple nucleotide repeats. ${ }^{13}$ The resulting microsatellite instability (MSI) is a characteristic of MMR deficiency and is found in the majority of CRCs arising in HNPCC. ${ }^{14}{ }^{15}$ To date, germline mutations affecting hMLHl, ${ }^{16}{ }^{17}$ hMSH2, ${ }^{18}{ }^{19}$ hPMS1, hPMS2, ${ }^{20}$ and hMSH $^{21}$ have been documented in HNPCC families.

Deficiency of MMR is also known to play a role in the development of CRC more generally. MSI is found in approximately $15 \%$ of all CRCs, ${ }^{13}{ }^{14}$ particularly those located in the proximal colon. ${ }^{22}$ The significance of MSI in such cancers is unclear. It may be assumed that some cases represent unrecognised HNPCC (that is, in association with germline mutations, even in the absence of a clear family history). However, in the majority of sporadic CRCs, MMR deficiency is thought to be an acquired event, resulting from somatic mutation or abnormal DNA methylation. ${ }^{23}{ }^{24}$ It is not clear whether the events leading to loss of MMR truly occur by chance or whether some individuals are susceptible to the development of such abnormalities. This study examines the

Abbreviations: MMR, mismatch repair; HNPCC, hereditary nonpolyposis colorectal cancer; CRC, colorectal cancer; $\mathrm{MSI}$, microsatellite instability; $O R$, odds ratio 
relationship between family history of cancer and development of MMR deficient CRC.

\section{METHOD}

\section{Study population}

Two CRC populations were identified. Appropriate ethics approval was obtained and members of each group provided informed consent for participation in this study. Characteristics of the study populations are detailed below.

\section{Hospital based group}

Individuals with histologically proven CRC were identified from the outpatient clinic of a single CRC surgeon. Patients diagnosed consecutively between 1987 and 1999 were approached to participate. If the affected patient was deceased, permission to include that patient in the study was sought from the next of kin.

\section{Population based group}

This group consisted of patients recruited to a multicentre case control study of diet. Eligible patients had histologically confirmed CRC reported to the Yorkshire Cancer Registry between September 1997 and October 1999. All cases were aged between 45 and 80 years, were resident within Leeds, and were treated within the Leeds Teaching Hospitals. The case control study excluded all individuals with a previous diagnosis of cancer, cancer arising in the context of familial polyposis coli, inflammatory bowel disease, or suggestive of metastatic spread.

\section{Assessment of family history}

After permission was obtained from their general practitioners and/or hospital consultants, individuals from the two CRC study groups were invited to attend for personal interview to assess family history. If the proband was deceased, next of kin were approached. Interviews were undertaken by trained interviewers, with emphasis placed on reports of cancer in first degree relatives (parents, siblings, and children). Particular attention was paid to diagnoses of HNPCC spectrum cancer (colon and rectum, stomach, small bowel, hepatobiliary tract, female reproductive tract, and urinary tract). Cancer diagnoses in relatives were verified against pathology reports, hospital notes, death certificates, or cancer registry records.

Demographic data (current age, age at diagnosis, sex) was recorded for all first degree relatives. Pedigrees were also assessed for features of dominantly inherited cancer susceptibility suggestive of HNPCC using the Amsterdam criteria (I and II) and modified ICG-HNPCC diagnostic criteria outlined in table 1. Details of family history in the hospital based group have been reported in part previously. ${ }^{25-27}$

\section{Immunohistochemical analysis}

Tissue ascertainment

Paraffin embedded tissue from the identified CRC was obtained for all patients. Tissue sections, each $4 \mu \mathrm{m}$, were selected for each tumour that contained both CRC tissue and normal colonic mucosa.

\section{Immunohistochemical assessment of hMLH 1 and hMSH2 expression}

Expression of hMLH1 and hMSH2 was assessed using an immunohistochemical technique reported previously. ${ }^{28}$ Tissue sections were deparaffinised with xylene and rehydrated through graded alcohols to water. Endogenous peroxidase was blocked using 3\% (v/v) hydrogen peroxide in methanol for 20 minutes. Antigenic site retrieval was achieved by boiling in 1.5 litres of $1: 100$ antigen unmasking solution (Vector Laboratories, Peterborough, UK) at $103 \mathrm{kPa}$ for 90 seconds. Subsequent immunohistochemical reactions were performed using the Sequenza system (Shandon, Basingstoke, UK). Non-specific antibody binding was prevented by incubation with $1 \%$ casein (Vector Laboratories) in Tris buffered saline ( $\mathrm{pH}$ 7.6) for 10 minutes. Endogenous biotin was then blocked using the avidin/biotin blocking kit (Vector Laboratories), each applied for 15 minutes. Tissue sections were then incubated with monoclonal hMSH2 antibody (Calbiochem, Nottingham, UK) or monoclonal hMLHI (Pharmingen, San Diego, California, USA) overnight at $4{ }^{\circ} \mathrm{C}$. Antibodies were made up in a solution of $0.2 \%$ casein in Tris buffered saline ( $\mathrm{pH} 7.6)$, obtaining a final dilution of 1:100 hMSH2 and 1:70 hMLH1. A third slide, not treated with either hMLH1 or hMSH2, served as a negative control. Tissue bound hMSH2 and hMLHl were identified by addition of a secondary antibody, using the StreptABComplex/HRP Duet Kit (Dako Ltd, High Wycombe, UK), and then visualised using $0.01 \%$ 3,3'-diaminobenzidine in Tris buffered saline. Diaminobenzidine staining was intensified using $0.5 \%$ copper sulphate in $0.9 \%$ saline for five minutes. Sections were then counterstained with haematoxylin.

\section{Assessment of MMR gene expression}

All tissue sections were examined using a light microscope for nuclear staining of hMLHl and hMSH2 protein. Expression was recorded as normal when nuclear staining was observed. Expression was considered abnormal when nuclear staining in cancer tissue was entirely absent in the presence of normal mucosal and/or lymphoid staining. Inclusion of normal mucosa in each tissue section thus served as an internal control. Staining was reviewed independently by LC or RC, and then re-reviewed jointly by LC and RC. Examples of staining patterns can be found elsewhere. ${ }^{28}$

\begin{tabular}{|c|c|c|c|}
\hline & Total & Hospital series & Population series \\
\hline $\begin{array}{l}\text { Total number of cases } \\
\text { Sex }\end{array}$ & 732 & $509(69.5)$ & $223(30.5)$ \\
\hline $\begin{array}{l}\text { Male (\%) } \\
\text { Female (\%) }\end{array}$ & $\begin{array}{l}417 \\
315\end{array}$ & $\begin{array}{l}285(56.0) \\
224(44.0)\end{array}$ & $\begin{array}{r}132(59.2) \\
91(40.8)\end{array}$ \\
\hline \multicolumn{4}{|c|}{ Age at diagnosis of CRC (y) } \\
\hline $\begin{array}{l}\text { Median } \\
\text { Range }\end{array}$ & $\begin{array}{l}68 \\
27-93\end{array}$ & $\begin{array}{l}69 \\
27-93\end{array}$ & $\begin{array}{l}67 \\
45-80\end{array}$ \\
\hline \multicolumn{4}{|l|}{ Tumour location } \\
\hline $\begin{array}{l}\text { Proximal (\%) } \\
\text { Distal (\%) }\end{array}$ & $\begin{array}{l}183(25.0) \\
549(75.0)\end{array}$ & $\begin{array}{l}127(25.0) \\
382(75.0)\end{array}$ & $\begin{array}{r}56(25.1) \\
167(74.9)\end{array}$ \\
\hline
\end{tabular}


Table 2 Family history characteristics of 732 patients presenting to the Leeds Teaching Hospitals with colorectal cancer, 1987-1999, with family history data and immunohistochemical results

\begin{tabular}{|c|c|c|c|c|}
\hline & Total & Hospital series & Population series & $\mathrm{p}$ Value \\
\hline FDR with CRC & $142(19.4)$ & $102(20.0)$ & $40(17.9)$ & $0.54^{*}$ \\
\hline \multicolumn{5}{|l|}{ No of affected FDRs } \\
\hline 0 & $590(80.6)$ & $407(80.0)$ & $183(82.1)$ & \\
\hline 1 & $121(16.5)$ & $88(17.3)$ & $33(14.8)$ & \\
\hline 2 or 3 & $21(2.9)$ & $14(2.7)$ & $7(3.1)$ & $0.39 \dagger$ \\
\hline FDR with extracolonic cancer* & 87 (11.9) & $65(12.8)$ & $22(9.9)$ & $0.32^{\star}$ \\
\hline \multicolumn{5}{|l|}{ No of affected FDRs } \\
\hline 0 & $645(88.1)$ & $444(87.2)$ & $201(90.1)$ & \\
\hline 1 & $76(10.4)$ & $56(11.0)$ & $20(9.0)$ & \\
\hline 2 or 3 & $11(1.5)$ & $9(1.8)$ & $2(0.9)$ & $0.63 † \ddagger$ \\
\hline \multicolumn{5}{|l|}{$\mathrm{FH}$ suggestive of HNPCC } \\
\hline Total & $19(2.6)$ & $14(2.7)$ & $5(2.2)$ & \\
\hline None & $713(97.4)$ & $495(97.3)$ & $218(97.8)$ & \\
\hline Amsterdam & $6(0.8)$ & $6(1.2)$ & $0(0)$ & \\
\hline Modified & $13(1.9)$ & $8(1.6)$ & $5(2.7)$ & $0.07 \bullet \ddagger$ \\
\hline \multicolumn{5}{|c|}{$\begin{array}{l}\text { CRC, colorectal cancer; FDR, first degree relative; HNPCC, hereditary non-polyposis colorectal cancer; } \mathrm{FH} \text {, family } \\
\text { history. } \\
\text { " } \chi \text { p palue compares reports of CRC and extracolonic cancer in relatives of CRC cases identified from hospital and } \\
\text { population series. } \\
\text { tp value compares number of CRC and extracolonic cancer cases between hospital and population series. } \\
\text { tp value from Fisher's exact test. } \\
\text { op value compares number of family history pedigrees meeting HNPCC criteria between study groups. }\end{array}$} \\
\hline
\end{tabular}

\section{Statistical analysis}

Differences in sex and tumour location and reported family history between the two study groups were compared using a two sided $\chi^{2}$ analysis and/or Fisher's exact test. Age at diagnosis was compared using the Kruskal-Wallis equality of populations test. The association between loss of expression of hMLHI and hMSH2 and family history of HNPCC spectrum cancer was assessed using single and multiple logistic regression. Initially the impact of each putative risk factor (for example, age, sex, anatomical location, family history) was assessed separately with logistic regression. As many of these risk factors are interrelated (for example, sex and anatomical location of tumour), we used multiple logistic regression to identify the independent effects of each putative risk factor. Odds ratios (OR) were used as measures of association, together with the associated 95\% confidence intervals (CI).

Relative risk estimates, together with the Poisson probability for the relative risk of colorectal and HNPCC spectrum extracolonic cancer in first degree relatives, were calculated by comparing the observed incidence of cancer in the study populations with an expected cancer incidence based on cancer incidence rates in the general population (obtained from the Yorkshire Cancer Registry for the years 1984-1986). Years at risk were determined from current age, age at death, or age at diagnosis of cancer.

Statistical significance was assumed where $p \leqslant 0.05$. All statistical analyses were performed using the Stata statistical software package (College Station, Texas, USA).

Table 3 Clinical criteria for suspicion of hereditary nonpolyposis colorectal cancer (HNPCC). ${ }^{39}$ Revised ICGHNPCC criteria (Amsterdam I and II)

There should be at least 3 relatives with CRC, or there should be at least 3 relatives with an HNPCC associated cancer (CRC, endometrium, small bowel, ureter, or renal pelvis); all the following should be present:

- One should be a first degree relative of the other two

- At least 2 successive generations should be affected

- At least 1 CRC should be diagnosed before the age of 50

- Familial adenomatous polyposis should be excluded

- Tumours should be verified by pathological examination

ICG, International Collaborative Group; CRC, colorectal cancer.

\section{RESULTS}

\section{Population demographics}

Hospital based CRC group

A total of 909 cases of CRC were identified in the hospital based group. All cases were diagnosed between 1987 and 1999. Of these, $644(70.8 \%)$ underwent interview to assess family history. Colorectal cancer tissue was obtained for 516 (80.1\% of those interviewed) cases with a known family history, and 509 were successfully stained for hMLHl and hMSH2.

\section{Population based case control group}

A total of 452 residents of Leeds diagnosed with CRC were reported during the recruitment phase of the case control study detailed above. Two hundred and three cases were subsequently found to be ineligible for further study on the basis of age, coexisting or previous disease, ethnic background, or declined the invitation to participate. The remaining 249 individuals comprise the population based case control group although 24 were already part of the hospital based case group and are included in that dataset for this analysis. All cases were aged $45-80$ years at the time of diagnosis. All cases underwent interview to assess family history. CRC tissue was obtained for the 226 remaining cases and $223(98.7 \%)$ were successfully stained for hMLHl and hMSH2.

Characteristics of the study groups are compared in table 1. Both groups were comparable for sex and tumour location. Thirty four $(5.3 \%)$ hospital based cases were found to have

Table 4 Criteria for suspicion of hereditary nonpolyposis colorectal cancer (HNPCC) based on ICG definition of HNPCC (Lynch syndrome)

Familial clustering of CRC and/or endometrial cancer (2 first degree relatives across 2 generations, with further affected second degree relatives)

- Associated cancers of: stomach, ovary, ureter/renal pelvis, small bowel, hepatobiliary tract, skin (sebaceous tumours)

- Development of cancer at an early age

- Development of multiple cancers, particularly multiple CRCs

- Proximal location of CRC

ICG, International Collaborative Group; CRC, colorectal cancer 
Table 5 Association between mismatch repair gene expression and family history of colorectal and extracolonic cancer based on univariate logistic regression

\begin{tabular}{|c|c|c|c|c|c|c|}
\hline & \multicolumn{3}{|c|}{ Loss of hMLHI } & \multicolumn{3}{|c|}{ Loss of hMSH2 } \\
\hline & ORt & $95 \% \mathrm{Cl} \dagger$ & p Valuet & ORt & $95 \% \mathrm{Cl} \dagger$ & $\mathrm{p}$ Valuet \\
\hline \multicolumn{7}{|l|}{ Colorectal cancer } \\
\hline FDR with CRC $\ddagger$ & 0.53 & $0.22-1.26$ & 0.15 & 1.05 & $0.12-9.45$ & 0.97 \\
\hline \multicolumn{7}{|l|}{ No of affected FDRs } \\
\hline $0 \ddagger$ & 1.00 & & & 1.00 & & \\
\hline 1 & 0.52 & $0.20-1.32$ & 0.17 & 0.00 & - & 0.37 \\
\hline 2 or 3 & 0.59 & $0.08-4.51$ & 0.61 & 7.34 & $0.78-68.66$ & 0.08 \\
\hline \multicolumn{7}{|l|}{ Extracolonic cancer } \\
\hline FDR with extracolonic cancer $¥$ & 0.96 & $0.40-2.33$ & 0.94 & 5.04 & $0.83-30.57$ & 0.08 \\
\hline \multicolumn{7}{|l|}{ No of affected FDRs } \\
\hline $0 \ddagger$ & 1.00 & & & 1.00 & & \\
\hline 1 & 0.54 & $0.16-1.76$ & 0.30 & 5.78 & $0.95-35.18$ & 0.06 \\
\hline 2 or 3 & 4.88 & $1.25-19.03$ & 0.02 & 0.00 & - & 0.82 \\
\hline \multicolumn{7}{|l|}{ FH suggestive of HNPCC } \\
\hline None & 1.00 & & & 1.00 & & \\
\hline Amsterdam (I and II) or modified & 1.56 & $0.35-6.94$ & 0.56 & 27.84 & $4.37-177.56$ & $<0.0001$ \\
\hline \multicolumn{7}{|c|}{$\begin{array}{l}\text { OR, odds ratio; } \mathrm{Cl} \text {, confidence interval; } \mathrm{CRC} \text {, colorectal cancer; FH, family history; FDR, first degree relative; } \\
\text { HNPCC, hereditary non-polyposis colorectal cancer. } \\
\text { *FH of HNPCC spectrum extracolonic cancer. } \\
\text { tOdds ratios, confidence intervals, and p values refer to loss of expression of hMLH1 and hMSH2 compared with } \\
\text { findividuals with no affected FDR. }\end{array}$} \\
\hline
\end{tabular}

synchronous tumours or occurred in individuals previously affected by a primary HNPCC spectrum cancer. Median age at the time of diagnosis of CRC was lower in the population based group by 1.3 years ( $p=0.07$, Kruskal-Wallis equality of populations test). This reflects exclusion of individuals over 80 years of age in the population based group.

\section{Family history characteristics}

Information was obtained regarding 4824 first degree relatives. Of these, $683(\%)$ were reported to have been affected by cancer and 207 (38.1\%) of these cancers were from the HNPCC spectrum. One hundred and eighty eight $(69.9 \%)$ were subsequently confirmed against the cancer register, case notes, death certificates, or pathology records. There was no difference in age at diagnosis between the HNPCC spectrum cancer group and the confirmed cancer subgroup (mean ages 66.0 and 65.9 years, respectively (range 27-95); $\mathrm{p}=0.53$ ).

Family history characteristics for the study groups are summarised in table 2 . There were no significant differences in family history between the two study groups. Family history of CRC (first degree relatives only) was reported in $20.0 \%$ and $17.9 \%$ of patients in the hospital based and population based groups, respectively (test of equality, $\mathrm{p}=0.52$ by $\chi^{2}$ test). First degree relatives affected by HNPCC spectrum extracolonic cancer were reported in $12.8 \%$ of hospital based and $9.8 \%$ of population based patients (test of equality, $\mathrm{p}=0.25$ by $\chi^{2}$ test). Overall, a first degree relative affected by CRC was reported by $19.3 \%$ of the combined hospital and population based groups while $11.9 \%$ reported a first degree relative affected by extracolonic cancer. There was one individual with more than two first degree relatives with CRC while one patient $(0.2 \%)$ reported three first degree relatives affected by extracolonic cancer. Nineteen patients $(2.6 \%)$ gave a family history that was suggestive of HNPCC. Of these, six $(0.8 \%)$ met the Amsterdam criteria while $13 \quad(1.8 \%)$ met the revised Amsterdam (II) criteria or were suspicious of HNPCC using modified diagnostic criteria.

\section{hMLH 1 and hMSH2 expression in CRC}

hMLH1 and hMSH2 expression was assessed in a total of 732 CRCs from the combined study groups. Six hundred and seventy five $(92.0 \%)$ CRCs exhibited normal expression of both hMLHI and hMSH2. Of the 57 (8.0\%) remaining CRCs, $52(7.1 \%)$ showed no staining for hMLHl while just five $(0.9 \%)$ demonstrated loss of hMSH2. Loss of expression was similar in both study groups (Fisher's exact test, $\mathrm{p}=1.0$ ). Using univariate logistic regression, loss of expression of hMLHl in CRC tissue was associated with sex (male compared with female: OR 0.49 (95\% CI $0.27-0.86$ ); $\mathrm{p}=0.01$ ) and proximal location (proximal compared with distal: OR 25.02 (95\% CI 11.04-56.69); $p<0.0001$ ). A second HNPCC spectrum cancer was associated with an OR of 2.38 (95\% CI $0.88-6.42 ; p=0.09$ ). Loss of hMLHl was more common with increasing age at diagnosis (OR 1.05 (95\% CI 1.02-1.08); $\mathrm{p}=0.001$ for each increasing year). Loss of expression of hMSH2 was non-significantly associated with female sex (OR 0.18 (95\% CI 0.02-1.68); p=0.13) and the presence of a second HNPCC spectrum cancer (OR 32.8 (95\% CI 7.03-153.11); $\mathrm{p}<0.0001$ ). All hMSH2 deficient CRCs were located in the proximal colon. In contrast with hMLHl deficient tumours, loss of hMSH2 was less common with increasing age (OR 0.93 (95\% CI 0.87-1.00); $\mathrm{p}=0.06$ for each increasing year).

\section{Amsterdam criteria and loss of expression}

Nineteen cases had a family history consistent with one of the definitions of HNPCC in tables 3 and 4. Of these, two (10.5\%) had loss of expression of hMSH2 and two (10.5\%) of hMLH1. All four of the tumours were right sided. The two cases with hMSH2 loss of expression each had a second HNPCC primary (renal pelvis and ovary) plus a family history of CRC and endometrial cancer. One case with hMLHl loss of expression had a family history of CRC, stomach cancer, and pancreatic cancer while the second had a family history of stomach cancer, pancreatic cancer, and endometrial cancer.

\section{Family history and hMLH 1 and hMSH2 expression}

Of the five cases with loss of expression for hMSH2, two had a family history satisfying the modified Amsterdam criteria (see previous section). For hMLHl, nine of the 52 (17.3\%) cases with loss of expression had a family history compared with 196 of $484(28.8 \%)$ of those without loss of expression $(p=0.07$ for equal proportions). Family history characteristics of MMR proficient and hMLH1 and hMSH2 deficient CRC are summarised in tables 5-7. Extent of family history was assessed as any first degree relative with cancer and 
Table 6 Association between mismatch repair gene expression and family history of colorectal and extracolonic cancer based on multivariate logistic regression

\begin{tabular}{|c|c|c|c|c|c|c|}
\hline & \multicolumn{3}{|c|}{ Loss of hMLH1§ } & \multicolumn{3}{|c|}{ Loss of hMSH2ף } \\
\hline & ORt & $95 \% \mathrm{Cl} \dagger$ & p Valuet & ORt & $95 \% \mathrm{Cl}+$ & p Valuet \\
\hline \multicolumn{6}{|l|}{ No of affected FDR } & 0.23 \\
\hline $0 \ddagger$ & 1.00 & & & 1.00 & & \\
\hline 1 & 0.46 & $0.16-1.29$ & 0.14 & - & - & - \\
\hline 2 or 3 & 0.55 & $0.06-5.14$ & 0.60 & 0.40 & $0.02-9.79$ & 0.57 \\
\hline \multicolumn{6}{|l|}{ No of affected FDR } & 0.49 \\
\hline $0 \ddagger$ & 1.00 & & & 1.00 & & \\
\hline 1 & 0.61 & $0.16-2.27$ & 0.46 & 2.59 & $0.27-24.89$ & 0.41 \\
\hline 2 or 3 & 9.15 & $1.18-70.84$ & 0.03 & - & - & - \\
\hline \multicolumn{7}{|c|}{$\begin{array}{l}\text { OR, odds ratio; } \mathrm{Cl} \text {, confidence interval; CRC, colorectal cancer; FH, family history; FDR, first degree relative. } \\
\text { *FH of HNPCC spectrum extracolonic cancer. } \\
\text { †Odds ratios, confidence intervals, and p values refer to loss of expression of hMLH1 and hMSH2 compared with } \\
\text { findividuals with no affected FDR. } \\
\text { ๆAdjusted for age at diagnosis, sex, and family history suggestive of HNPCC (not adjusted for tumour location as } \\
\text { all tumours were right sided). } \\
\text { §Adjusted for tumour location, age at diagnosis, sex, and family history suggestive of HNPCC. }\end{array}$} \\
\hline
\end{tabular}

number of affected first degree relatives. Table 5 summarises odds ratios for MMR deficient CRC based on family history of colorectal and HNPCC spectrum extracolonic cancer using univariate logistic regression. Table 6 summarises odds ratios for MMR deficient CRC using a multivariate logistic regression analysis. Table 7 summarises relative cancer risk in first degree relatives of patients with MMR proficient and hMLHl and hMSH2 deficient CRC.

In table 5, there was no association between loss of hMLHl or hMSH2 and family history of CRC overall $(\mathrm{p}=0.15$ for hMLH1, $\mathrm{p}=0.97$ for hMSH2). In the presence of multiple affected relatives, this comparison approached significance for hMSH2 (OR 7.34 (95\% CI 0.78-68.7); p =0.08). For extracolonic tumours, there was no association with loss of expression of hMLHl $(\mathrm{p}=0.94)$ except for the category of two or more affected relatives (OR 4.88 (95\% CI 1.25-19.03); $\mathrm{p}=0.02$ ). For hMSH2, the risk for those with a family history approached significance (OR 5.04 (95\% CI $0.83-30.57$ ); $\mathrm{p}=0.08$ ); this effect was all due to the family history of one extracolonic cancer.

The association between hMLH1 deficient CRC and family history of HNPCC extracolonic cancer was seen to be more marked using a multivariate logistic regression analysis (including adjustment for tumour location, sex, age at diagnosis, and family history suggestive of HNPCC: OR 9.15 (95\% CI 1.18-70.84); $\mathrm{p}=0.03$ ) (table 6). This association was seen whether or not HNPCC type family history was adjusted for (data not shown). These findings suggest that the observed association between family history of HNPCC spectrum extracolonic cancer and hMLHI deficient CRC is independent of clinically recognisable HNPCC based on family history assessment. However, the effect of family history on hMSH2 loss of expression in multivariate analysis was reduced (from that in the univariate analysis) after adjusting for a family history suggestive of HNPCC.

Assessment of family history using Amsterdam criteria (I and II) or modified Amsterdam criteria identified that while loss of hMSH2 was clearly associated with a family history suggestive of HNPCC (OR 27.84 (95\% CI 4.37-177.56); $\mathrm{p}<0.0001)$, such a history was not predictive of loss of hMLHI (OR 1.56 (95\% CI 0.35-6.94); p=0.56).

Table 7 summarises relative cancer risk in first degree relatives of CRC cases. Relatives were seen to be at increased risk of developing CRC (relative risk 1.63, p<0.0001). Cancers of the stomach, ovary, uterus, and pancreas were seen to occur at the same incidence as in the reference population. Relative cancers risks were seen to be similar in relatives of individuals with MMR proficient CRC. Relatives of individuals with hMSH2 deficient CRC were at increased risk of uterine cancer (relative risk 25.0, p=0.003). Although relative risk of CRC was also raised (relative risk 2.70), this was not statistically significant. Relatives of individuals with hMLHl deficient CRC were not seen to be at increased risk of any cancer.

\section{DISCUSSION}

This study has detailed the family history characteristics of a large cohort of CRC patients. This cohort was of significance in that cases were identified consecutively and, we believe, can be assumed to be representative of the CRC population in general. Accordingly, CRC cases included in this study were predominantly distal in location, were equally distributed by sex, and occurred at a median age of 70 years.

Familial clustering of CRC is a consistent finding in epidemiological studies. ${ }^{429}$ Although it is recognised that recall of family events can be unreliable, ${ }^{31} 70 \%$ of cancers considered of interest in this study were subsequently confirmed against cancer registry or hospital records. However, reports of other cancers outside the HNPCC spectrum were less reliable, with only $39 \%$ confirmed. This may, in part, reflect a bias towards confirmation of those cancers considered significant in the setting of familial CRC. There has been less attention paid in the literature to the association between CRC and family history of extracolonic cancer. There is evidence that close relatives of individuals with CRC are also at increased risk of developing cancer of the uterus, ovary, stomach, and breast. ${ }^{32-34}$ In this study, HNPCC spectrum extracolonic cancer was reported in $5.1 \%$ of first degree relatives.

The incidence of HNPCC in the general population is difficult to estimate. While generally quoted as 5\% of all CRCs, numerous studies estimate the HNPCC prevalence to be much lower. ${ }^{35}{ }^{36}$ In this study, HNPCC assessed by family history alone was estimated to be $2.6 \%$. Six families $(0.8 \%)$ met the Amsterdam criteria (I and II) while a further 13 ( $1.8 \%$ ) were thought to represent HNPCC based on modified criteria.

Germline mutations in DNA MMR genes play a causative role in the development of CRC through HNPCC. Modelling has suggested that the familial risk is not due entirely to HNPCC, ${ }^{37}$ raising the possibility that MMR abnormalities play a broader role in susceptibility. Alternatively, acquired mutations may be more common in certain individuals rendered susceptible, perhaps by the interaction of inherited 
Table 7 Association between expression of $h M L H 1$ and $h M S H 2$ and relative risk of cancer in first degree relatives of patients with colorectal cancer

\begin{tabular}{|c|c|c|c|c|c|}
\hline $\begin{array}{l}\text { MMR status in } \\
\text { proband }\end{array}$ & $\begin{array}{l}\text { Tumour site } \\
\text { in FDR }\end{array}$ & $\begin{array}{l}\text { No of observed } \\
\text { cancers }\end{array}$ & $\begin{array}{l}\text { No of expected } \\
\text { cancers }\end{array}$ & RR & p Value* \\
\hline \multirow[t]{8}{*}{ All CRCs } & Stomach & 50 & 42.1 & 1.18 & 0.13 \\
\hline & Pancreas & 13 & 21.1 & 0.61 & - \\
\hline & Uterus & 14 & 16.0 & 0.88 & - \\
\hline & Ovary & 10 & 20.3 & 0.49 & - \\
\hline & Gall b́ladder & 4 & 3.8 & 1.04 & 0.53 \\
\hline & Kidney & 5 & 14.7 & 0.34 & - \\
\hline & Colon'rectum & 164 & 100.52 & 1.63 & $<0.0001$ \\
\hline & HNPCC sites & 260 & 218.4 & 1.19 & 0.003 \\
\hline \multirow[t]{8}{*}{ MMR competent† } & Stomach & 47 & 38.7 & 1.21 & 0.11 \\
\hline & Pancreas & 11 & 19.3 & 0.57 & - \\
\hline & Uterus & 10 & 14.6 & 0.68 & - \\
\hline & Ovary & 9 & 18.6 & 0.48 & - \\
\hline & Gall b́ladder & 3 & 3.5 & 0.85 & - \\
\hline & Kidney & 5 & 13.5 & 0.37 & - \\
\hline & Colon/rectum & 155 & 92.2 & 1.68 & $<0.0001$ \\
\hline & HNPCC sites & 240 & 200.3 & 1.19 & 0.004 \\
\hline \multirow[t]{8}{*}{ hMSH2 deficient } & Stomach & - & - & - & - \\
\hline & Pancreas & - & - & - & - \\
\hline & Uterus & 2 & 0.08 & 25.00 & $<0.0001$ \\
\hline & Ovary & - & - & - & - \\
\hline & Gall b́ladder & - & - & - & - \\
\hline & Kidney & - & - & - & - \\
\hline & Colon / rectum & 2 & 0.74 & 2.70 & 0.17 \\
\hline & HNPCC sites & 4 & 1.54 & 2.59 & 0.07 \\
\hline \multirow[t]{8}{*}{ hMLHI deficient } & Stomach & 3 & 3.12 & 0.96 & 0.56 \\
\hline & Pancreas & 2 & 1.59 & 1.26 & 0.47 \\
\hline & Uterus & 2 & 1.3 & 1.54 & 0.14 \\
\hline & Ovary & 1 & 1.6 & 0.64 & - \\
\hline & Gall bladder & 1 & 0.3 & 3.33 & 0.26 \\
\hline & Kidney & - & - & - & - \\
\hline & Colon/rectum & 7 & 7.6 & 0.92 & 0.20 \\
\hline & HNPCC sites & 16 & 16.5 & 0.97 & 0.40 \\
\hline \multicolumn{6}{|c|}{$\begin{array}{l}\text { MMR, mismatch repair; FDR, first degree relative; RR, relative risk; HNPCC, hereditary non-polyposis colorectal } \\
\text { cancer. } \\
\text { " } \mathrm{p} \text { values for RR>1 only; } \\
+ \text { TNormal expression of } h M L H 1 \text { and } h M S H 2 \text {. } \\
\text { HNPCC sites include stomach, pancreas, uterus, ovary, colon, rectum, kidney, and aall bladder }\end{array}$} \\
\hline
\end{tabular}

polymorphic genes and environmental exposure. Eight per cent of cancers in this study were shown to be deficient in MMR. A small proportion of these cancers could represent HNPCC cases but there was no evidence of an association between loss of MMR and family history of CRC except for multiple affected relatives and hMSH2. These findings are in keeping with previous studies. ${ }^{27}{ }^{38}$ In comparison, however, there is evidence of an association between MMR deficient CRC and family history of HNPCC spectrum extracolonic cancer. Loss of hMLH1 and hMSH2 was approximately five times more common in individuals with a family history of extracolonic cancer (of two or three relatives for hMLHl or one relative for hMSH2), compared with those with no family history. The general characteristics of hMSH2 deficient CRC (proximal location, young age at diagnosis, association with development of multiple primary cancers, and association with family history suggestive of HNPCC) are consistent with causative germline mutations. hMLHI deficient CRCs identified in this study do not exhibit characteristics suggestive of HNPCC. In contrast with hMSH2 deficient cancer, hMLHl deficient CRCs tend to occur at a later age ( 75.0 years of age for those with a family history of colorectal or HNPCC spectrum) and in the absence of a family history suggestive of HNPCC. The observed association between hMLHl deficient CRC and family history of HNPCC spectrum extracolonic cancer persists with or without inclusion of HNPCC-like characteristics in a multivariate analysis, suggesting that loss of hMLHI in CRC occurs independently of germline MMR mutations. These data therefore suggest another mechanism predisposing to loss of hMLHI in individuals with a family history of extracolonic cancer. An example of such a putative mechanism would be susceptibility to promoter methylation as methylation is known to be associated with loss of expression of hMLHl. ${ }^{23}{ }^{24}$ Such an observation of promoter hypermethylation of hMLHl has been made for adenomatous polyps found in close relatives of patients with CRC. ${ }^{40}$

\section{ACKNOWLEDGMENTS}

We wish to thank Sally Gray, Department of Histopathology; Leeds Teaching Hospitals NHS Trust, and the Northern and Yorkshire Cancer Registry and Information Service (NYCRIS) for their assistance in this study. RC was funded by the Leslie Silver Charitable Trust. LC, SB, and PQ were funded by Yorkshire Cancer Research. This research was supported by Cancer Research UK.

\section{Authors' affiliations}

R P Coggins, Cancer Research UK Genetic Epidemiology Laboratory, St James's University Hospital, Leeds, and Department of Surgery, Leeds General Infirmary, Leeds, UK

G P Crockford, D T Bishop, Cancer Research UK Genetic Epidemiology Laboratory, St James's University Hospital, Leeds, UK

P J Finan, Department of Surgery, Leeds General Infirmary, Leeds L Cawkwell, S M Bell, P Quirke, Academic Unit of Pathology, University of Leeds, Leeds

Conflict of interest: None declared.

\section{REFERENCES}

1 Duncan JL, Kyle J. Family incidence of carcinoma of the colon and rectum in north-east Scotland. Gut 1982;23:169-71.

2 Kune GA, Kune S, Watson LF. The role of heredity in the etiology of large bowel cancer: data from the Melbourne Colorectal Cancer Study. World I Surg 1989; 13:124-9.

3 Johns LE, Houlston RS. A systematic review and meta-analysis of familial colorectal cancer risk. Am J Gastroenterol 2001;96:2992-3003. 
4 St John DJ, McDermott FT, Hopper JL, et al. Cancer risk in relatives of patients with common colorectal cancer. Ann Intern Med 1993;1 18:785-90.

5 Fuchs CS, Giovannucci EL, Colditz GA, et al. A prospective study of family history and the risk of colorectal cancer. N Engl J Med 1994;331:1669-74

6 Bonelli L, Martines H, Conio M, et al. Family history of colorectal cancer as a risk factor for benign and malignant tumours of the large bowel. A casecontrol study. Int J Cancer 1988;41:513-17.

7 Bazzoli F, Fossi S, Sottili S, et al. The risk of adenomatous polyps in asymptomatic first-degree relatives of persons with colon cancer. Gastroenterology 1995;109:783-8.

8 Lynch HT, Smyrk T. Hereditary nonpolyposis colorectal cancer (Lynch syndrome). An updated review. Cancer 1996;78:1149-67.

9 Chung DC, Rustgi AK. DNA mismatch repair and cancer. Gastroenterology 1995; 109:1685-99

10 Jiricny J. Mediating mismatch repair. Nat Genet 2000;24:6-8.

11 Lipkin SM, Wang V, Jacoby R, et al. MLH3: a DNA mismatch repair gene associated with mammalian microsatellite instability. Nat Genet 2000;24:27-35.

12 Malkhosyan S, Rampino N, Yamamoto H, et al. Frameshift mutator mutations. Nature 1996;382:499-500.

13 lonov Y, Peinado MA, Malkhosyan S, et al. Ubiquitous somatic mutations in simple repeated sequences reveal a new mechanism for colonic carcinogenesis. Nature 1993;363:558-61.

14 Aaltonen LA, Peltomaki P, Leach FS, et al. Clues to the pathogenesis of familial colorectal cancer. Science 1993;260:812-16.

15 Aaltonen LA, Peltomaki P, Mecklin J-P, et al. Replication errors in benign and malignant tumors from hereditary nonpolyposis colorectal cancer patients. Cancer Res 1994:54:1645-8.

16 Papadopoulos N, Nicolaides NC, Wei Y-F, et al. Mutation of a mutL homolog in hereditary colon cancer. Science 1994;263:1625-9.

17 Bronner CE, Baker SM, Morrison PT, et al. Mutation in the DNA mismatch repair gene homologue $\mathrm{hMLH} 1$ is associated with hereditary non-polyposis colon cancer. Nature 1994;368:258-61.

18 Fishel R, Lescoe MK, Rao MR, et al. The human mutator gene homolog MSH2 and its association with hereditary nonpolyposis colon cancer. Cell 1993;75:1027-38.

19 Leach FS, Nicolaides NC, Papadopoulos N, et al. Mutations of a mutS homolog in hereditary nonpolyposis colorectal cancer. Cell 1993;75:1215-25.

20 Nicolaides NC, Papadopoulos N, Liu B, et al. Mutations of two PMS homologues in hereditary nonpolyposis colon cancer. Nature 1994:371:75-80.

21 Akiyama Y, Sato H, Yamada T, et al. Germ-line mutation of the hMSH6/ GTBP gene in an atypical hereditary nonpolyposis colorectal cancer kindred. Cancer Res 1997; 57:3920-3.

22 Thibodeau SN, Bren G, Schaid D. Microsatellite instability in cancer of the proximal colon. Science 1993;260:816-19.

23 Cunningham JM, Christensen ER, Tester DJ, et al. Hypermethylation of the hMLH1 promoter in colon cancer with microsatellite instability. Cancer Res 1998;58:3455-60.
24 Herman JG, Umar A, Polyak K, et al. Incidence and functional consequences of hMLH1 promoter hypermethylation in colorectal carcinoma. Proc Natl Acad Sci U S A 1998;95:6870-5.

25 Stephenson BM, Finan PJ, Gascoyne J, et al. Frequency of familial colorectal cancer. Br J Surg 1991;78:1162-6.

26 Hall NR, Bishop DT, Stephenson BM, et al. Hereditary susceptibility to colorectal cancer. Relatives of early onset cases are particularly at risk. Dis Colon Rectum 1996;39:739-43.

27 Brown SR, Finan PJ, Cawkwell L, et al. Frequency of replication errors in colorectal cancer and their association with family history. Gut 1998;43:553-7.

28 Cawkwell L, Gray S, Murgatroyd H, et al. Choice of management strategy for colorectal cancer based on a diagnostic immunohistochemical test for defective mismatch repair. Gut 1999;45:409-15.

29 Macklin MT. Inheritance of cancer of the stomach and large intestine in man. J Natl Cancer Inst 1960;24:551-71.

30 Kune GA, Kune S, Warson LF. The role of heredity in the etiology of large bowel cancer: Data from the Melbourne Colorectal Cancer Study. World J Surg 1989;13:124-31.

31 Aitken J, Bain C, Ward M, et al. How accurate is self-reported family history of colorectal cancer? Am J Epidemiol 1995;141:863-71.

32 Nelson CL, Sellers TA, Rich SS, et al. Familial clustering of colon, breast, uterine, and ovarian cancers as assessed by family history. Genet Epidemiol 1993; 10:235-44.

33 Gruber SB, Thompson WD. A population-based study of endometrial cancer and familial risk in younger women. Cancer and Steroid Hormone Study Group. Cancer Epidemiol Biomarkers Prev 1996;5:41 1-17.

34 Slattery ML, Kerber RA. Family history of cancer and colon cancer risk: the Utah Population Database. J Natl Cancer Inst 1994;86:1618-26.

35 Mecklin J-P, Jarvinen HJ, Hakkiluoto A, et al. Frequency of hereditary nonpolyposis colorectal cancer: A prospective multicenter study in Finland. Dis Colon Rectum 1995;38:588-93.

36 Evans DG, Walsh S, Jeacock J, et al. Incidence of hereditary non-polyposis colorectal cancer in a population-based study of 1137 consecutive cases of colorectal cancer. Br J Surg 1997;84:1281-5.

37 Jenkins MA, Baglietto L, Dite GS, et al. After hMSH2 and hMLH1 - what next? Analysis of three-generational, population-based, early-onset colorectal cancer families. Int J Cancer 2002;102:166-71

38 Samowitz WS, Slattery ML, Kerber RA. Microsatellite instability in human colonic cancer is not a useful clinical indicator of familial colorectal cancer. Gastroenterology 1995; 109:1765-71.

39 Vasen HFA, Watson P, Mecklin J-P, et al. New clinical criteria for hereditary nonpolyposis colorectal cancer (HNPCC, Lynch syndrome) proposed by the International Collaborative Group on HNPCC. Gastroenterology 1999; 116:1453-6.

40 Ricciardiello L, Goel A, Mantovani V, et al. Frequent loss of hMLHI by promoter hypermethylation leads to microsatellite instability in adenomatous polyps of patients with a single first-degree member affected by colon cancer. Cancer Res 2003;63:787-92. 